\title{
Refractory Acute Kidney Injury Secondary to Hypothyroid- Induced Myopathy: A Contemporary Case Report
}

\author{
Phillip Ulyanovskiya, b, Deborah Dergan ${ }^{\mathrm{a}}$, Shams Abbas ${ }^{\mathrm{a}}$, Prabhjot Manes ${ }^{\mathrm{a}}$, \\ Irina Erlikh ${ }^{\mathrm{a}}$
}

\begin{abstract}
This is a case report elucidating how severe hypothyroid-induced myopathy can lead to refractory kidney injury, unmanageable by standard medical and hemodialysis interventions. A 70-year-old female with extensive medical comorbidities including adult onset hypothyroidism, hypertension, hyperlipidemia, and normal baseline renal function presented with shortness of breath, myalgias, edema, and facial swelling. The patient was found to have a thyroid-stimulating hormone of $169.8 \mu \mathrm{IU} / \mathrm{mL}$, creatine phosphokinase of $42,670 \mathrm{U} / \mathrm{L}$, blood urea nitrogen of $70 \mathrm{mg} / \mathrm{dL}$, a creatinine of $12.1 \mathrm{mg} / \mathrm{dL}$, and glomerular filtration rate of $3 \mathrm{~mL} / \mathrm{min} / 1.73 \mathrm{~m}^{2}$. She was initiated on aggressive intravenous isotonic rehydration, along with intensive intravenous thyroid hormone replacement therapy and hydrocortisone treatment as well. Her renal status failed to improve adequately and she was started on sodium bicarbonate for urinary alkalinization. With the preceding interventions deemed medically futile for renal amelioration, the patient was started on acute hemodialysis. Over the course of 2 weeks and six hemodialysis treatments, the patient's renal status failed to improve. The patient finally refused any further hemodialysis or medical interventions seeing that her kidneys failed to respond to treatment, and her clinical prognosis remained poor. This case report illustrates how severe symptomatic hypothyroidism can induce rhabdomyolysis leading to intractable kidney failure, unmanageable by standard medical therapy and hemodialysis.
\end{abstract}

Keywords: Hypothyroidism; Hypothyroid-induced myopathy; Rhabdomyolysis; Acute kidney injury; Acute renal failure; Acute hemodialysis; Thyroid hormone; Thyroid hormone replacement therapy

\section{Introduction}

Myopathy can be one of the clinical features of hypothyroidism. Multiple studies have suggested a possible link between

Manuscript accepted for publication November 30, 2016

aDepartment of Family Medicine, The Brooklyn Hospital Center, Icahn School of Medicine at Mount Sinai, 121 Dekalb Avenue, Brooklyn, NY 11201, USA ${ }^{b}$ Corresponding Author: Phillip Ulyanovskiy, Department of Family Medicine, The Brooklyn Hospital Center, Icahn School of Medicine at Mount Sinai, 121 Dekalb Avenue, Brooklyn, NY 11201, USA.

Email: phil.ulyanovskiy@gmail.com

doi: https://doi.org/10.14740/jmc2699w impairment in mitochondrial metabolism due to decreased phosphocreatine in chronic hypothyroidism and subsequent elevation in a creatine kinase indicating muscle destruction [13]. It is common to see muscular dysfunction in the setting of hypothyroidism $[4,5]$. Some instances have even evolved into serious complications like acute kidney injury secondary to rhabdomyolysis [6-9]. Several case reports have documented an uncommon correlation between hypothyroidism and associated acute renal failure. However, only a handful of these instances have revealed extremely critical states in which muscle tissue destruction ensued to a point where hormone readministration, hydration, and hemodialysis failed to clinically improve renal status and patient recovery. We report one such case of severe hypothyroidism with associated rhabdomyolysis leading to acute kidney failure, wherein thyroid hormone replacement therapy and acute hemodialysis failed to resolve kidney function.

\section{Case Report}

A 70-year-old woman who presented with a 4-day history of progressively worsening shortness of breath was admitted to our institution. She also noted generalized myalgia, weakness, and edema with evident facial swelling. The patient additionally complained of left-sided, intermittent, non-radiating chest pain with 5/10 intensity and associated orthopnea. Her prior medical history comprised of hypertension, hyperlipidemia, coronary artery disease status post coronary artery bypass graft, adult onset hypothyroidism, cervical spinal stenosis, history of transient ischemic attack (date unknown), chronic obstructive pulmonary disease/well-controlled intermittent asthma, uncontrolled type 2 diabetes mellitus with diabetic neuropathy, Parkinson's disease, and vascular dementia. Her home medication regimen included aspirin $81 \mathrm{mg}$ daily, carvedilol $6.25 \mathrm{mg}$ twice daily, Detemir 52 units nightly, Januvia $100 \mathrm{mg}$ daily, insulin aspart 14 units three times daily, melatonin, lisinopril $5 \mathrm{mg}$ daily, and synthroid $150 \mu \mathrm{g}$ daily. The patient was noted to be poorly compliant with her medications and overall medical care.

On physical examination, the patient was obese and exhibited facial edema as well as bilateral lower extremity pitting edema. On admission, her laboratory tests were notable for aspartate aminotransferase (AST) $>717 \mathrm{U} / \mathrm{L}$, alanine aminotransferase $(\mathrm{ALT})>227 \mathrm{U} / \mathrm{L}$, thyroid-stimulating hormone (TSH) of $169.8 \mu \mathrm{IU} / \mathrm{mL}, \mathrm{T} 3<1.0$, free $\mathrm{T} 4<0.4$, bicarbonate level of $15 \mathrm{mmol} / \mathrm{L}$, blood urea nitrogen $(\mathrm{BUN})$ of $70 \mathrm{mg} / \mathrm{dL}$, 
creatinine of $12.1 \mathrm{mg} / \mathrm{dL}$, and glomerular filtration rate (GFR) of $3 \mathrm{~mL} / \mathrm{min} / 1.73 \mathrm{~m}^{2}$. Her baseline levels of BUN, Cr, and GFR were $11-17 \mathrm{mg} / \mathrm{dL}, 0.9 \mathrm{mg} / \mathrm{dL}$, and $62 \mathrm{~mL} / \mathrm{min} / 1.73 \mathrm{~m}^{2}$, respectively. The patient's CKMB on admissions was 237.2 $\mathrm{ng} / \mathrm{mL}$ and creatine phosphokinase (CPK) was 42,670 U/L.

Troponin was negative when evaluated on three different occasions and separated by an 8-h time span. Additionally, EKG demonstrated sinus rhythm at a rate of $63 \mathrm{bpm}$, left axis deviation, and non-specific ST changes. Her chest radiograph displayed hyperinflated lungs compatible with chronic obstructive pulmonary disease, normal heart size, and status post coronary bypass graft with sternal wires and mediastinal clips.

The patient was diagnosed with severe symptomatic hypothyroidism with rhabdomyolysis and acute kidney failure. Endocrinology was consulted and the patient was initiated on $100 \mathrm{mg}$ hydrocortisone via IVPB with re-administration of levothyroxine $100 \mathrm{mg}$ IVPB and later advanced to $150 \mu \mathrm{g}$. Initially, due to the patient's acute kidney failure, nephrology recommended adequate hydration with isotonic IV fluids. However, acute hemodialysis was deemed necessary owing to the patient's poor urine output $(<400 \mathrm{~mL}$ produced in any $24-\mathrm{h}$ period). A non-tunneled hemodialysis catheter was therefore placed for subsequent treatment. Fluid infusion rate was decreased to avoid volume overload despite the patient's oliguric state, and she was also given sodium bicarbonate to alkalinize her urine. Over the course of treatment, the patient was placed on calcitriol with supplemental calcium.

During her hospital stay, the patient received six dialysis treatments over the course of approximately 14 days. She was noted to have refused dialysis early in her care despite lengthy discussions of how this decision could greatly impact her state of health and prognosis. Nevertheless, after receiving aggressive treatments including dialysis sessions, IV fluids, IV levothyroxine, and hydrocortisone, the patient's creatine kinase decreased from 42,670 to 1,582 U/L, serum $\mathrm{CO}_{2}$ improved from 12 to $22 \mathrm{mmol} / \mathrm{L}, \mathrm{BUN}$ improved from 70 to $43 \mathrm{mg} / \mathrm{dL}$, and $\mathrm{Cr}$ improved from 12.1 to $8.5 \mathrm{mg} / \mathrm{dL}$. Despite improvement in $\mathrm{CK}, \mathrm{CO}_{2}, \mathrm{BUN}$, and $\mathrm{Cr}$, the patient's GFR remained relatively unchanged throughout hospitalization, only improving just slightly from 3 to $5 \mathrm{~mL} / \mathrm{min} / 1.73 \mathrm{~m}^{2}$. Ultimately, the patient did not wish to continue with dialysis treatments and agreed to comfort care only via hospice.

\section{Discussion}

Muscle involvement is commonly found in adults who suffer from hypothyroidism, and is more likely to be found in those with severe or longstanding untreated hypothyroidism [3]. Patients with hypothyroidism frequently have elevated creatine kinase $[1,3]$. This marker for muscular damage can often be elevated for years before clinically evident features of hypothyroidism emerge. Furthermore, myalgia, in addition to muscle hypertrophy and proximal myopathy, is also observed in adults with hypothyroidism. Various case reports have noted very high serum creatine kinase and rhabdomyolysis associated with hypothyroid disease $[3,5,6]$. In some cases, vigorous exercise or concurrent statin therapy administration also seems to precipitate rhabdomyolysis. Rarely, rhabdomyolysis secondary to hypothyroidism can culminate into dangerous complications such as renal failure $[7,9]$. Thus, patients reporting fatigue or myalgia require further evaluation by measuring variations in their TSH level. Additionally, electromyography can be useful in determining whether muscle involvement is secondary to hypothyroidism as opposed to inflammatory myositis. Invasive diagnostic procedures like muscle biopsy are not typically necessary.

The pathogenesis of hypothyroid myopathy is poorly understood. Symptoms of muscle involvement and abnormalities in muscle function may possibly be related to the effects of hypothyroidism on cellular metabolism [2]. Thyroxine (T4) affects energy metabolism. Deficient levels of T4 can lead to abnormalities of glycogenolysis, mitochondrial oxidative metabolism, and triglyceride turnover, all of which can impact muscle function [10]. Additionally, studies have linked a deficiency in skeletal muscle L-carnitine found in hypothyroid patients, which subsequently normalizes upon establishing a euthyroid state via hormone replacement [11].

As previously indicated, hypothyroid myopathy is treated by replacement of thyroid hormone. Hormone replacement therapy usually lowers creatine kinase levels, which typically return to baseline over a few weeks, indicating decreased muscle destruction $[3,11]$. TSH levels, however, usually require an extended period of time to normalize [12]. With regard to prognosis, notwithstanding the normalization in creatine kinase and TSH levels within weeks to months, prospective analyses have indicated that actual functional muscle strength improvement requires over a year of hormone treatment along with physiotherapy $[4,13]$. Initial recovery required 3.6 months of therapeutic intervention, while residual weakness resolved after a year of treatment [4]. Thus, usually with prompt diagnosis and treatment, patients have a good prognosis. However, in rare cases such as the one described, individuals who are refractory to therapy might require renal replacement or terminal palliative care.

\section{Conflicts of Interest}

None.

\section{References}

1. Chavez LO, Leon M, Einav S, Varon J. Beyond muscle destruction: a systematic review of rhabdomyolysis for clinical practice. Crit Care. 2016;20(1):135.

2. Kaminsky P, Robin-Lherbier B, Brunotte F, Escanye JM, Walker P, Klein M, Robert J, et al. Energetic metabolism in hypothyroid skeletal muscle, as studied by phosphorus magnetic resonance spectroscopy. J Clin Endocrinol Metab. 1992;74(1):124-129.

3. Scott KR, Simmons Z, Boyer PJ. Hypothyroid myopathy with a strikingly elevated serum creatine kinase level. Muscle Nerve. 2002;26(1):141-144.

4. Duyff RF, Van den Bosch J, Laman DM, van Loon BJ, Linssen WH. Neuromuscular findings in thyroid dysfunc- 
tion: a prospective clinical and electrodiagnostic study. J Neurol Neurosurg Psychiatry. 2000;68(6):750-755.

5. Siafakas NM, Salesiotou V, Filaditaki V, Tzanakis N, Thalassinos N, Bouros D. Respiratory muscle strength in hypothyroidism. Chest. 1992;102(1):189-194.

6. Altay M, Duranay M, Ceri M. Rhabdomyolysis due to hypothyroidism. Nephrol Dial Transplant. 2005;20(4):847848.

7. Barahona MJ, Mauri A, Sucunza N, Paredes R, Wagner AM. Hypothyroidism as a cause of rhabdomyolysis. Endocr J. 2002;49(6):621-623.

8. Bosch X, Poch E, Grau JM. Rhabdomyolysis and acute kidney injury. N Engl J Med. 2009;361(1):62-72.

9. Kisakol G, Tunc R, Kaya A. Rhabdomyolysis in a patient with hypothyroidism. Endocr J. 2003;50(2):221-223.

10. Giampietro O, Clerico A, Buzzigoli G, Del Chicca MG,
Boni C, Carpi A. Detection of hypothyroid myopathy by measurement of various serum muscle markers - myoglobin, creatine kinase, lactate dehydrogenase and their isoenzymes. Correlations with thyroid hormone levels (free and total) and clinical usefulness. Horm Res. 1984;19(4):232-242.

11. Sinclair C, Gilchrist JM, Hennessey JV, Kandula M. Muscle carnitine in hypo- and hyperthyroidism. Muscle Nerve. 2005;32(3):357-359.

12. Morris JC. How do you approach the problem of TSH elevation in a patient on high-dose thyroid hormone replacement? Clin Endocrinol (Oxf). 2009;70(5):671-673.

13. Sharma V, Borah P, Basumatary LJ, Das M, Goswami M, Kayal AK. Myopathies of endocrine disorders: A prospective clinical and biochemical study. Ann Indian Acad Neurol. 2014;17(3):298-302. 